\title{
CE-OFDM-CDMA Phase Modulation for 5G System
}

\author{
Jamal Mestoui \\ ISNT Team, IMAGE Laboratory, \\ Moulay Ismail University of Meknes, Morocco. \\ Corresponding author: j.mestoui@edu.umi.ac.ma \\ Mohammed El Ghzaoui \\ Faculty of Sciences, \\ Sidi Mohamed Ben Abdellah University, Fes, Morocco. \\ E-mail: elghzaoui.mohammed@gmail.com

\section{Serghini Elaage} \\ ISNT Team, IMAGE Laboratory, \\ Moulay Ismail University of Meknes, Morocco. \\ E-mail: elaages@gmail.com \\ Abdelmounim Hmamou \\ TTI Team, ERTTI Laboratory, \\ Moulay Ismail University of Meknes, Morocco. \\ E-mail: hmamou0512@gmail.com

\section{Jaouad Foshi} \\ TTI Team, ERTTI Laboratory, \\ Moulay Ismail University of Meknes, Morocco. \\ E-mail: j.foshi@yahoo.fr
}

(Received on February 10, 2021; Accepted on May 27, 2021)

\begin{abstract}
In the present work, we propose a novel modulation scheme for $5 \mathrm{G}$ wireless communication system. Our contribution is to combine PM-OFDM (Phase Modulation Orthogonal Frequency Division Multiplexing) and CDMA (Code Division Multiple Access) to exploit their distinctive advantages. On the one hand, PM-OFDM is an effective technique to combat multipath fading effects. On the other hand, CDMA can serve multiple users who are using the same resources of time/frequency. The aim is to make a combination of PM-OFDM and CDMA techniques. In this paper, the OFDM-CDMA scheme and its PAPR (Peak-to-Average Power Ratio) statistics are reviewed. In this paper, the proposed scheme PM-OFDM-CDMA is described and its performances in terms of PAPR, power spectral density, and BER (Bit Error Rate) are analyzed. Moreover, MMSE (Minimum Mean Square Error) equalizer is used to avoid multipath and noise effects simultaneously. The simulation through AWGN (Additive white Gaussian noise) and Rayleigh channels is performed using MATLAB. From the simulation results, we observed that PM-OFDM-CDMA is an efficient technique in terms of energy consumption (PAPR $=0 \mathrm{~dB}$ ). Besides, CE-OFDM-CDMA offers high spectral efficiency with low BER due to its low PAPR. In CE-OFDM-CDMA method, the shape of the spectrum varies according to the value of the modulation index $h$. The band occupied by the spectrum increases with the value of $h$. Therefore, CE-OFDM-CDMA could be considered as a suitable technique for 5G applications.
\end{abstract}

Keywords- PAPR, Energy efficiency, 5G, PM-OFDM-CDMA, BER. 


\section{Introduction}

Recently, technology has made huge steps, especially in the field of the development of mobile electronic devices. To support, the advanced requirements of high throughput. Future mobile communication systems must combine different modulation and multiple access techniques.

OFDM is a basic multi-carrier technique in the implementation of wireless communication networks. OFDM was a key solution in the development of the most important physical layer protocols: Wi-Fi "IEEE 802.11", LTE "3GPP", and Wi-MAX "IEEE 802.16" (Ansar and Noor, 2018). However, OFDM signals suffer from a high PAPR (Rajasekaran et al., 2019). The high PAPR associated with OFDM signal is caused by the power amplifier (PA) at the transmitter (Banelli, 2003). If any additional power is not injected in Input, OFDM suffers from spectral widening which causes by the non-linear component of the transceiver (Singya et al., 2017). This problem limits the performance of the OFDM system especially in the case of battery-powered wireless systems. These problems can be solved by increasing the IBO (Raab et al., 2002). Many technics have been suggested to resolve this problem (Mohammady et al., 2019, 2020). However, the PAPR reduction technics proposed in the literature suffer from either complexity or high BER (El Ghzaoui et al., 2020). The phase modulator has the advantage of producing a modulated signal with the lowest possible PAPR $(0 \mathrm{~dB})$. In this case, the PA can be used efficiently in the transmission chain based on the OFDM system. A zero PAPR allows the power amplifier to be used with maximum efficiency as well as increases the choice of the system as more signal power is radiated into the channel. On the other hand, the generations of wireless networks implemented so far have employed CDMA as a multi-user technique. In CDMA, each user is assigned a specific code for the duration of the call. For single-user technics, the capacity is restricted by the time/frequency resources. In CDMA technique, the number of users is fixed by the properties of the utilized spreading codes. Therefore, CDMA has the ability to increase the capacity of a wireless communication system.

5G communications systems affect virtually every fringe of society. This rapid expansion of telecommunications technology systems has been accompanied by increasing demands in terms of reliability or quality, transmission rate, mobility and flexibility, and this with increasingly miniaturized systems that generally have low energy resources. The OFDM present in several telecommunications standards is today the modulation technique to best meet these requirements. However, this technique presents high PAPR at the transmitter. This high PAPR reduces the energy efficiency of the power amplifier at emission. Several solutions allowing on the one hand to optimize the energy efficiency of the amplifier and on the other hand to minimize distortions due to its non-linearities have been suggested by researchers. Due to the characteristics of the power amplifier, optimizing the efficiency requires operation as close as possible to its saturation zone. And as we mentioned earlier, operating the power amplifier in this area causes distortions in the signal and these distortions are accentuated when the input signal presents high PAPR. Reducing the variations of the complex envelope of the input signal appears as a natural solution to combine energy efficiency and distortion mitigation. In other words, reducing the PAPR of the input signal is a solution allowing on the one hand to enhance the efficiency of the power amplifier and on the other hand to minimize the distortions induced by the power amplifier. In this paper, to reduce the PAPR associated with the OFDM technique we use constant envelop modulation instead of conventional OFDM. Moreover, other technics were proposed in the literature for $5 \mathrm{G}$ system. Combining OFDM and CDMA is also proposed for $5 \mathrm{G}$ applications in form of MC-CDMA, MC-DS-CDMA, and MT-CDMA (Judson et al., 2019). In (Judson et al., 2019), the performance of MC-CDMA in wireless channels is analyzed. The characteristics of 
MC-CDMA signal are studied by mean of Mean Peak Signal to Noise Ratio (PSNR) criterion. Moreover, MC-CDMA is implemented in an FPGA (Nahar et al., 2017). Furthermore, MCCDMA can be the best candidate for the physical layer in 5G (Razavi et al., 2017). In (Maatouk et al., 2018), the authors proposed an interesting NOMA architecture for 5G cellular networks which combines OFDMA with MC-CDMA. In this scheme, OFDMA sub-carriers are initially used, and when these sub-carriers are fully loaded, besides, other users will have access to the network using MC-CDMA. However, the major drawback of MC-CDMA is the high (PAPR) in OFDM which causes high power consumption, which is unattractive for battery-powered mobile communication systems. Besides, the CDMA suffers from interference and spectral regrowth when the number of users becomes important, so it is necessary to use either coding or estimation techniques or equalization to combat the interference. On our side, we propose a PM-OFDMCDMA as a suitable waveform. Compared to the proposed conventional waveforms for the $5 \mathrm{G}$ application, the PM-OFDM-CDMA system has better overall performance for future mobile communication networks. In addition to the multi-user aspect considered by the CDMA technique, its PAPR is OdB. So, the proposed scheme offers high capacity with low power consumption.

\section{Related Works}

A comparison between several $5 \mathrm{G}$ waveforms, such as OFDM, UFMC, FBMC, and GFDM, is made in terms of power spectral density, PAPR, and robustness of multi-user asynchronous uplink transmission (Gerzaguet et al., 2017). In (Van Eeckhaute et al., 2017), an extensive comparison of FBMC, GFDM, UFMC, and RB-F-OFDM in 5G context is performed. The performances of the proposed waveform were, compared to OFDM. It has been demonstrated that GFDM is the most suitable waveform for the 5G application. However, the majority of these waveforms suffer from PAPR. To overcome this problem Taspinar \& Simsir (Taspinar and Simsir, 2020) propose an efficient Migrating Birds Optimization-Selective Mapping (MBOSLM) technique to reduce the PAPR associated with the UFMC waveform. The MBO-SLM technique provided good performance compared to the conventional SLM method due to the optimization process in MBO-SLM. Lastly, many researches have been done to determine the suitability of the UFMC waveform for the 5G application (Schaich et al., 2014). However, it has been proofed in (Vakilian et al., 2013) that UFMC is the suitable waveform for short-burst communication. In (Baig et al., 2020) the cyclic prefix-based OFDM wave form has been proposed. A new wave form (DFT-s-OFDM) similar to the cyclic prefix-based OFDM waveform, has been proposed to support the $5 \mathrm{G}$ requirements (Baig et al., 2020).

\section{Conventional MC-CDMA System}

In this system, two important techniques in wireless communications are combined. The first is the CDMA multiple access methods, which are used to increase the capacity of the system by permitting to several users to take part in the same time and frequency resources. The second technique is OFDM modulation; this latter is used to increase the data rate. OFDM signal provides optimal use of the frequency resources by fighting interferences. Also, the problem of multipath is reduced by introducing redundancy codes.

\subsection{MC-CDMA System Model}

The MC-CDMA signal can be generated by the transmitter represented in Figure 1. The frequency-domain spreader is applied. The signal used by one user is separated from the signal of other users by the spreading sequences. These codes must be orthogonal. The Walsh Hadamard and gold codes are recommended for the implementation of the MC-CDMA scheme. 
The signal at the transmitter signal for the u-th user is given by:

$x_{M C}^{u}(t)=\sum_{k=0}^{N_{s u b}-1} d_{i}^{u} C S_{k}^{u} e^{2 \pi\left(f_{0}+k \Delta t\right)} \delta(t-i T)$

where $N_{\text {sub }}$ is the number of subcarriers, $d_{i}^{u}$ is the $i$-th data symbol of the $u$-th user, $f_{k}=f_{0}+k \Delta f$ is a frequency of $k$-th subcarrier, $f_{0}$ is the fundamental transmission frequency, and $\Delta f=1 / T$ is the space between two adjusted subcarriers.

$C S_{k}^{u},\left(\mathrm{k}=0,1, \ldots N_{s u b}-1\right)$ is the code sequence of the $u$-th user and $\delta(\mathrm{t})$ is a rectangular pulse filter defined by:

$\delta(t)=\left\{\begin{array}{cc}1 & 0 \leq t<T \\ 0 & \text { otherwise }\end{array}\right.$

At the receiver showing in Figure 2, and after the serial-to-parallel conversion, each carrier's symbol $d_{i}^{u}$ is recuperated by using FFT and the recuperated sequence is connected with the desired user's spreading code $C S_{k}^{u}$. Then, the equalization operation and other treatments are applied.

The $i$-th received sequence at the $k$-th subcarrier is defined by:

$y_{k, i}=\sum_{u=0}^{N_{u}} h_{k}^{u} C S_{k}^{u} d_{i}^{u}+w_{k, i}$

where $N_{u}$ is the number of users, $h_{k}^{u}$ is the channel transfer function of the $k$-th carrier and $w_{k, i}$ is the channel noise.

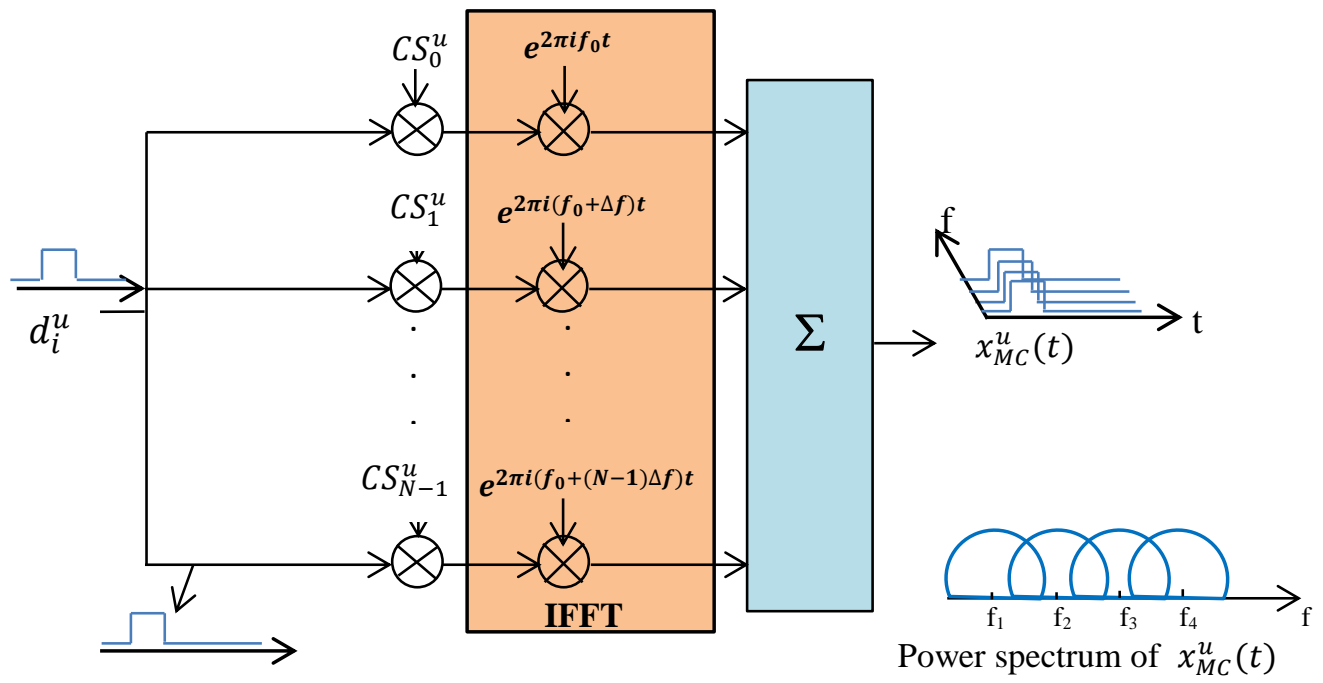

Figure 1. Conventional MC-CDMA transmitter. 


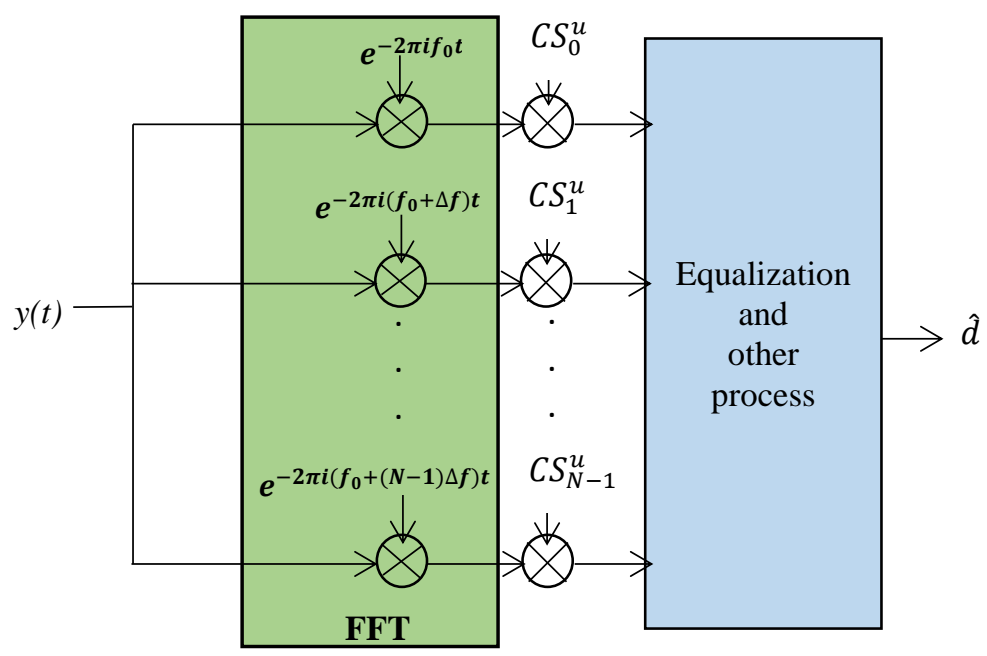

Figure 2. MC-CDMA receiver.

\subsection{PAPR Statistics of Conventional MC-CDMA}

MC-CDMA systems generally suffer from high PAPR. The high PAPR can reduce the spectral efficiency of the system as well as the efficiency of the power amplifiers used in mobile devices (Kumar and Venkatesan, 2020). Also, the interference between users, ICI, and the noise has a strong effect that weakens the spectral efficiency of MC-CDMA systems (Ahmed, 2019). In general, a high PAPR degrades the characteristics of the MC-CDMA system (Jangalwa and Tokekar, 2019).

Mathematically, the PAPR at the transmitter is given by:

$\operatorname{PAPR}\left(x_{M C}^{u}(t)\right)=\frac{\max _{0 \leq t<T}\left[\left|x_{M C}^{u}(t)\right|^{2}\right]}{E\left[\left|x_{M C}^{u}(t)\right|^{2}\right]}$

where $\max _{0 \leq t<T}\left[\left|x_{M C}^{u}(t)\right|^{2}\right]$ present peak power and $E\left[\left|x_{M C}^{u}(t)\right|^{2}\right]$ present average power of the clipped signal $x_{M C}^{u}(t)$.

The CCDF of PAPR is the most used function in the literature to measure the performance of MC-CDMA technique. The CCDF function can be represented by:

$C C D F_{P A P R\left(x_{M C}^{u}(t)\right)}=\operatorname{Pr}\left[\operatorname{PAPR}\left(x_{M C}^{u}(t)\right)>P A P R_{0}\right]$

where $P A P R_{0}$ is the threshold of the PAPR.

In practice, the calculation of the peak is performed on the discrete-time version of $x_{M C}^{u}(t)$ obtained by oversampling the expression described in Eq. (4). 
$\operatorname{PAPR}\left(x_{M C}^{u}[n]\right)=\frac{\max _{0 \leq t<T}\left[\left|x_{M C}^{u}[\ddot{n} n]\right|^{2}\right]}{E\left[\left|x_{M C}^{u}[n]\right|^{2}\right]}$

In Figure 3, the values of $P A P R_{0}$, at $\mathrm{CCDF}=10^{-2}$, are $6.6 \mathrm{~dB}$ and $8.4 \mathrm{~dB}$ for 16 and 32 subcarriers respectively. The results show that the MC-CDMA system offers poor PAPR performance as the number of carriers increases. This is due to the superposition of the signals which causes significant power variations.

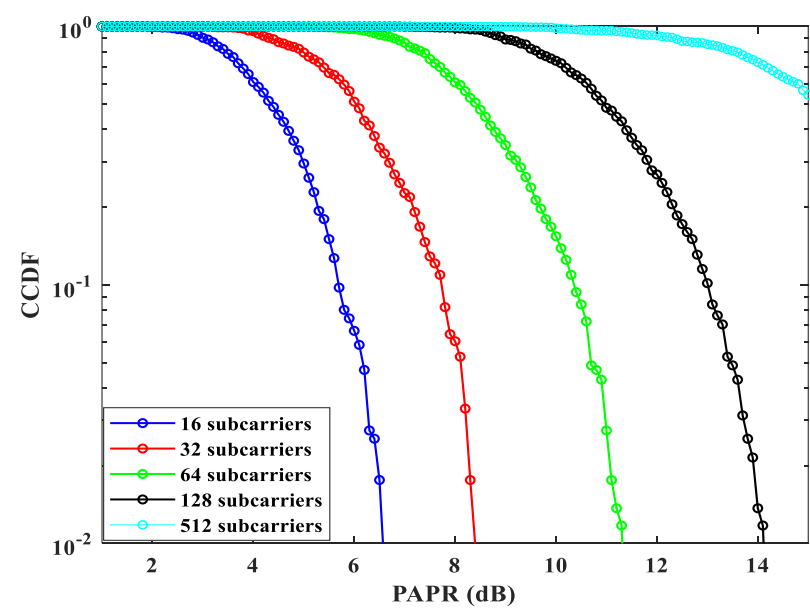

Figure 3. PAPR performance of MC-CDMA (Rayleigh channel).

\section{PM-OFDM-CDMA System}

OFDM-CDMA is a high power consumption technique, which is unwanted especially for batterypowered mobile communication systems. The PM-OFDM-CDMA system is a new version of the OFDM-CDMA system. PM-OFDM-CDMA system is multi-carrier modulation that uses OFDMCDMA with a phase modulator. In this way, the resulting signal is characterized by its constant amplitude. Therefore the PM-OFDM-CDMA system showing in Figure 4 has the lowest p PAPR.

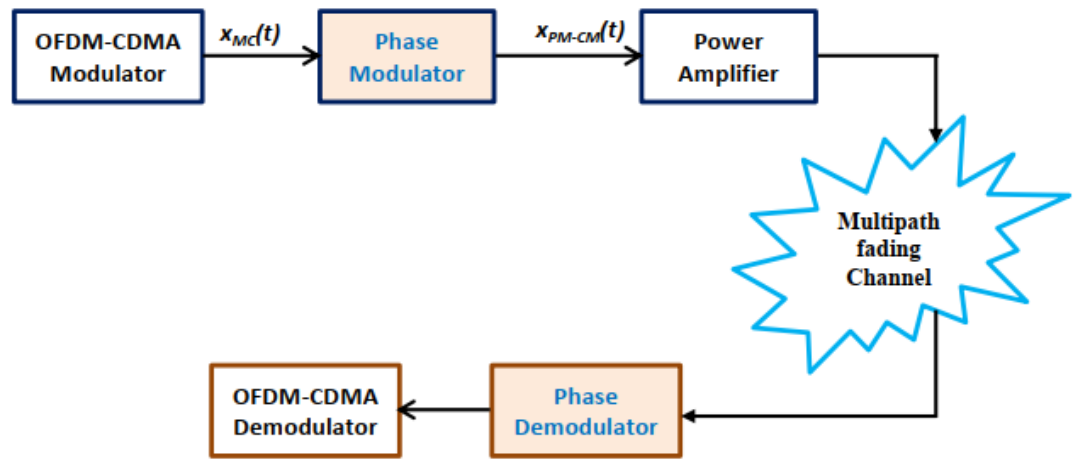

Figure 4. Base principle of the PM-OFDM-CDMA system. 
A transformation is applied to the resulting signal of Eq. (1) to modulate it in phase. In this way, we obtain a constant envelope waveform which is characterized by a reduced PAPR.

The $x_{M C}(t)$ signal is passed in a phase modulator to obtain the $x_{P M-M C}(t)$ signal. The signal at the output of the transmitter is written as follows:

$x_{P M-M C}(t)=A_{x} e^{j \beta(t)}$

where $A_{x}$ is the amplitude of the transmitted signal.

The phase signal during the i-th block is expressed:

$\beta(t)=\alpha_{i}+2 \pi h C_{s t} \sum_{k=1}^{N_{s u b}} d_{i, k}^{u} \operatorname{sub}\left(t-i T_{\text {OFDM }}\right) C S_{k}^{u}$

where $i T_{\text {OFDM }} \leq t<(i+1) T_{\text {OFDM }}, h$ represents the modulation index and $\alpha_{i}$ represents memory term. $C_{s t}$ is the normalizing constant which can be expressed by:

$C_{s t}=\sqrt{\frac{2}{N_{s u b} \sigma_{d}^{2}}}$

where $\sigma_{d}^{2}$ can be writing by the following equation:

$\sigma_{d}^{2}=E\left\{\left|d_{i, k}\right|^{2}\right\}=\frac{O_{M}-1}{3}$

this expression depends only on the order of modulation $O_{M}$. The energy of the signal at the transmitter is given by:

$\varepsilon_{x}=\int_{i T_{O F D M}}^{(i-1) T_{O F D M}}\left|x_{P M-M C}(t)\right|^{2} d t=A_{x}^{2}$

for bit energy we can use the following expression:

$\varepsilon_{b}=\frac{\varepsilon_{x}}{N_{\text {sub }} \log _{2} O_{M}}=\frac{A_{x}^{2}}{N_{\text {sub }} \log _{2} O_{M}}$

the data symbols that should be mapped are real-valued:

$d_{i, k} \in\left\{ \pm 1, \pm 3, \ldots, \pm\left(O_{M}-1\right)\right\}$

these amplitudes can be obtained by using the pulse-amplitude modulation (PAM). For CEOFDM technique used in this work we choose the subcarrier as:

$\operatorname{sub}_{k}(t)=\left\{\begin{array}{cl}\cos \left(\frac{2 \pi k t}{T_{\text {OFDM }}}\right), & 0 \leq t<T_{\text {OFDM }} \\ 0, & \text { otherwise }\end{array}\right.$ 

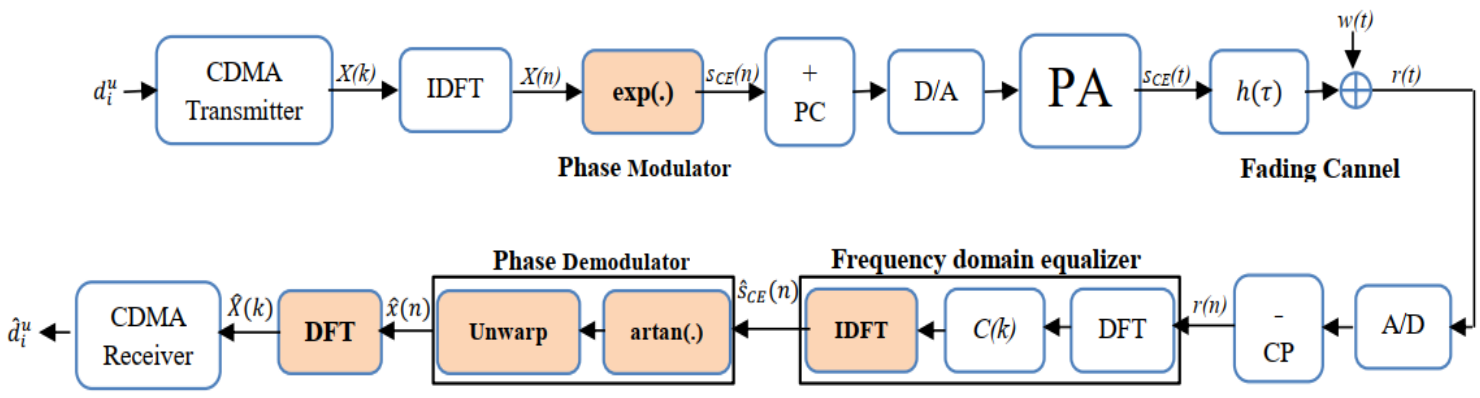

Figure 5. Baseband signal of a PM-OFDM-CDMA system.

In Figure 5, the blocks of the PM-OFDM-CDMA system are presented, $w(t)$ presents the additive noise. This system shares many practical blocks with classical MC-OFDM. In the detailed diagram of Figure 5, the data symbols are multiplied by a sequence of CDMA codes.

It is mentioned in the IEEE802.16.3 standard, if the cyclic prefix in a transmitted signal is greater than 20\%, the bandwidth allowed will be lost (Gupta and Tiwari, 2013). The PM-OFDM discretetime model and the MMSE equalizer described in (Mestoui et al., 2019) will be used in this work to analyze the performance of the proposed system. The baseband signal must be carried to the center frequency of the passband determined by the application before being transmitted. The signal transmitted, and then passed through a multipath fading channel, is picked up by the receiver. Removing the $\mathrm{CP}$ is the first step to be performed at the receiver after the received signal has been returned to baseband, the DFT of the signal is generated and frequency domain equalization is performed. The effect of the phase modulator is canceled by the following operations: IDFT in the FDE, arct(.) operation a phase unwrapped. An important remark is that phase demodulator receivers have the following characteristic: at low SNR the performance of the CE-OFDM system deteriorates considerably (Thompson, 2005).

The modulation without memory leads to spectral lines at frequencies $f_{k}=k / T_{\text {OFDM }}, k=(0, \pm 1$, $\pm 3, \ldots$ ) using the memory eliminates these lines. Different estimates can be used to determine the power spectral density of the CE-OFDM signal, such as Taylor's expansion and Abramson's spectrum (Thompson, 2005). The memory is defined by:

$\theta_{i}=\theta_{i-1}+K \sum_{k=1}^{N_{\text {sul }}}\left[d_{i, k} A_{b}(k)-d_{i-1, k} A_{e}(k)\right]$

The real bandwidth of the transmitted signal, defined as twice the highest frequency of $x_{M C}(t)$ is:

$B_{1}=2 \times \frac{N_{\text {sub }}}{2 T_{\text {OFDM }}}=\frac{N_{\text {sub }}}{T_{\text {OFDM }}}$

The more appropriate and conservative bandwidth of $x_{P M-M C}(t)$ is $B_{2}=\max (2 \pi h, 1) B_{1}$.

\section{Results and Discussion}

In this section, we discuss the obtained results. After building the Matlab model of the proposed PM-OFDM-CDMA transceiver, we have evaluated the performance of PM-OFDM-CDMA 
system with different values of modulation index $h$. We consider that the number of subcarriers is 64 , modulation order $M=4$ and the input data size amounts to $100.10^{6}$ source bits. The prototype filter is a shaping filter with a Hamming Window of length $=11$. Also, we used additive White Gaussian Noise (AWGN) as the channel noise and Rayleigh channel model to provide practical simulation in our simulation. The rest of the simulation parameters are summarized in the Table 1.

The results show that using a MMSE equalizer enables to obtain acceptable performance in terms of BER. In addition, we will compare the PM-OFDM-CDMA system with OFDM-CDMA in terms of PSD and BER.

Table 1. Simulation parameters.

\begin{tabular}{|c|c|c|c|c|c|}
\hline Parameter & Parameter description & Value & Parameter & Parameter description & Value \\
\hline \multicolumn{3}{|c|}{ OFDM-CDMA parameters } & \multicolumn{3}{|c|}{ PM-OFDM- CDMA parameters } \\
\hline$N$ & Number of subcarrier & 64 & $N$ & Number of subcarrier & 64 \\
\hline$N_{C P}$ & Cyclic Prefix length & $N / 4$ & $N_{C P}$ & Cyclic Prefix length & $N / 4$ \\
\hline numFFT & FFT size & 512 & $O_{f}$ & oversampling factor & 8 \\
\hline SNR & Signal-to-noise ratio & $0-45$ & numFFT & FFT size & $N * J$ \\
\hline & Code & Walsh Code & $2 \pi h$ & $\mathrm{~h}:$ modulation index & $0.2-1.2$ \\
\hline$L C S$ & Length of the code & 4 & SNR & Signal-to-noise ratio & $0-45$ \\
\hline
\end{tabular}

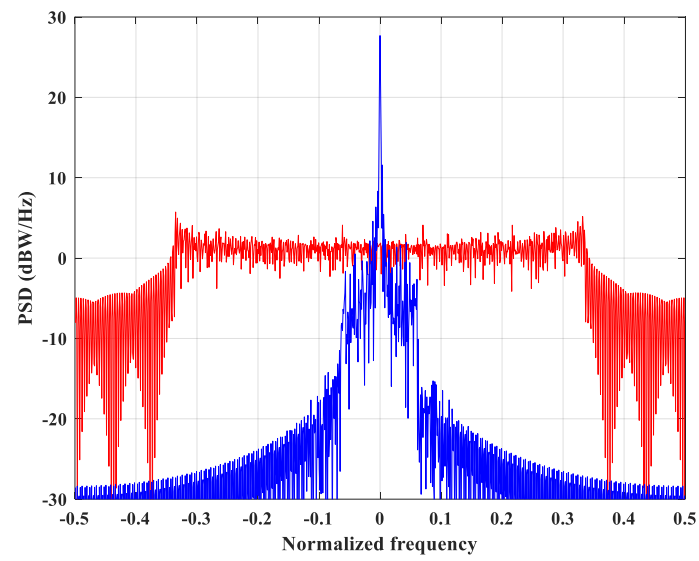

Figure 6. PSD for PM-OFDM-CDMA compared to OFDM-CDMA $(2 \pi \mathrm{h}=0.2)$.

Figures 6,7 and 8 show the spectral energy efficiency for different modulation index values $(2 \pi h=0.2,2 \pi h=0.6$ and $2 \pi h=1.2)$. For the OFDM-CDMA scheme, the energy spectrum is the same in all cases. Therefore, the PSD for this scheme is not controllable. In PM-OFDM-CDMA method, the shape of the spectrum varies according to the value of the modulation index. The band occupied by the spectrum increases with the value of $h$. 


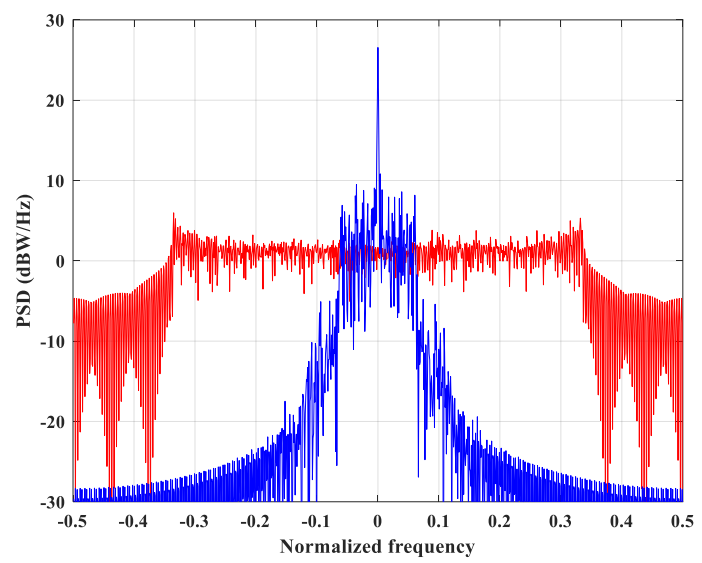

Figure 7. PSD for PM-OFDM-CDMA compared to OFDM-CDMA $(2 \pi \mathrm{h}=0.6)$.

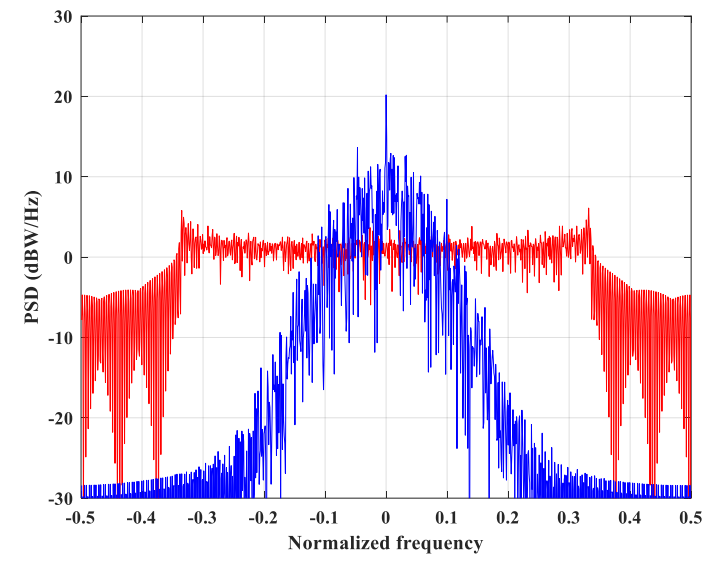

Figure 8. PSD for PM-OFDM-CDMA compared to OFDM-CDMA $(2 \pi \mathrm{h}=1.2)$.

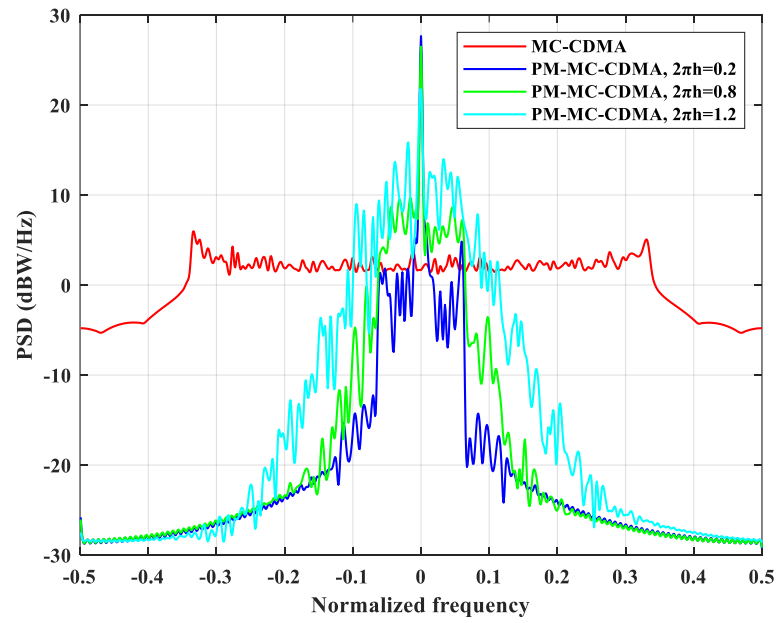

Figure 9. PSD envelope comparison. 
We observed from Figure 9 that the modulation index affect the CE-OFDM spectral confinement. A narrower spectrum is required when the value of $h$ is smaller. The trade-off when $h$ is smaller, the BER performance result is poorer. Consequently and according to the needs, the designer of the system can exchange the performances against the spectral confinement and inversely. PMOFDM's phase-continuous design exhibits faster side lobe roll-off than conventional OFDM.

The performance of the PM-OFDM-CDMA technique was evaluated on AWGN and Rayleigh fading channels. We have simulated and examined the performance of the system and the effect of code length Walsh Hadamard on these performances. The BER results are reported in Figures 10, 11, and 12. At the first stage, the performance of the proposed PM-OFDM-CDMA system is evaluated by AWGN channel conditions.

In Figure 10, the performances in terms of BER for the PM-OFDM-CDMA system for $2 \pi \mathrm{h}=0.2$, $0.4,0.6$ and 0.8 are shown. Wa can noted that the performance of the system can be enhanced by usinghigh modulation index h. For example, to have a BER of $10^{-3}$ we need a bit energy per error equal to $20.9 d B$ for $2 \pi h=0.2$, while for $2 \pi \mathrm{h}=0.8$ we only have $9.35 d B$.

In Figure 11 we evaluated the performance of a PM-OFDM-CDMA system model with $O_{M}=4$ and varying h over Rayleigh channel. At SNR per bit $E b / N O=20 \mathrm{~dB}$, if $2 \pi h=0.2$, the BER=0.02 and if $2 \pi h=1.0$, the $\mathrm{BER}=0.0006$. These results show that for the same value of $E b / N O=20 \mathrm{~dB}$, the system is more efficient in terms of BER when the values of $h$ used are large.

Figure 12 illustrates the simulation results of the PM-OFDM-CDMA system for different values of code length. In this work, $N_{\text {sub }}$ is set to be equal to the length of the Walsh Hadamard code. From the above results, we can observe that the PM-OFDM-CDMA system performs better in term of BER when the code length set to be 4 and 16. The performance of the proposed system is evaluated also for two values of modulation index $\mathrm{h}(2 \pi h=0.2$ and $2 \pi h=1.0)$. It can be observed that the BER of the system is better when using longer code sequences. As a result, the proposed system can be suitable for $5 \mathrm{G}$ applications.

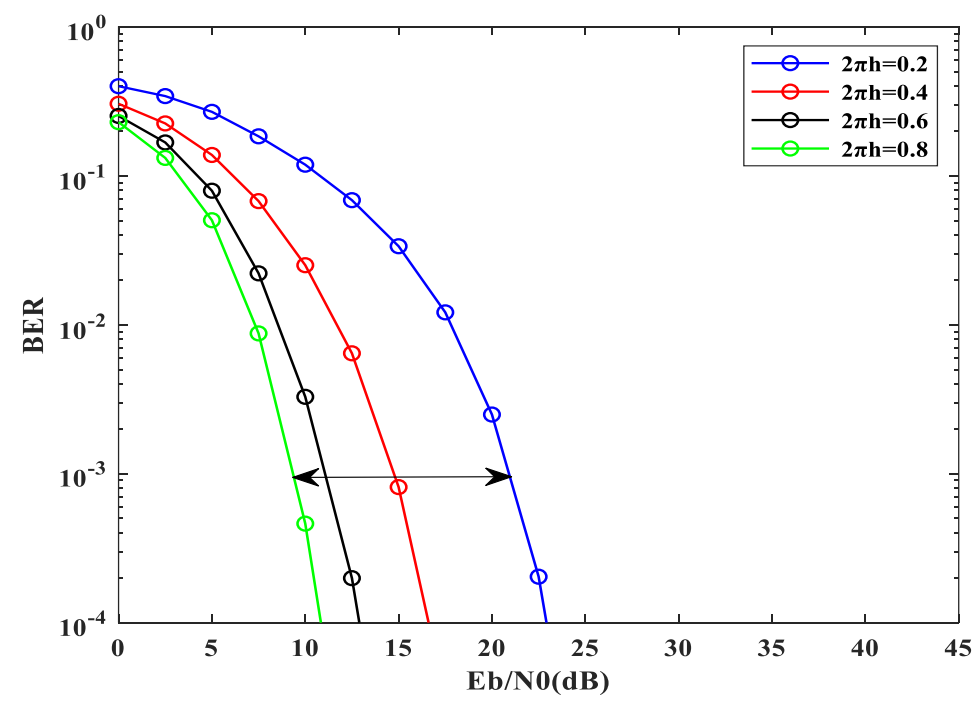

Figure 10. BER versus SNR of PM-OFDM-CDMA (AWGN channel). 


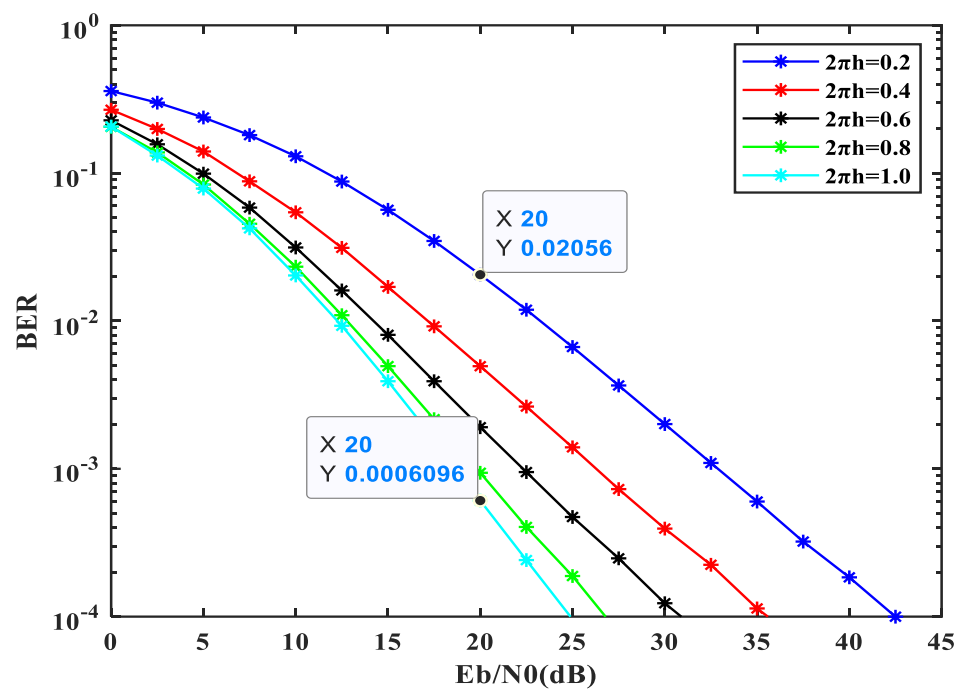

Figure 11. BER versus SNR of PM-OFDM-CDMA (Rayleigh channel).

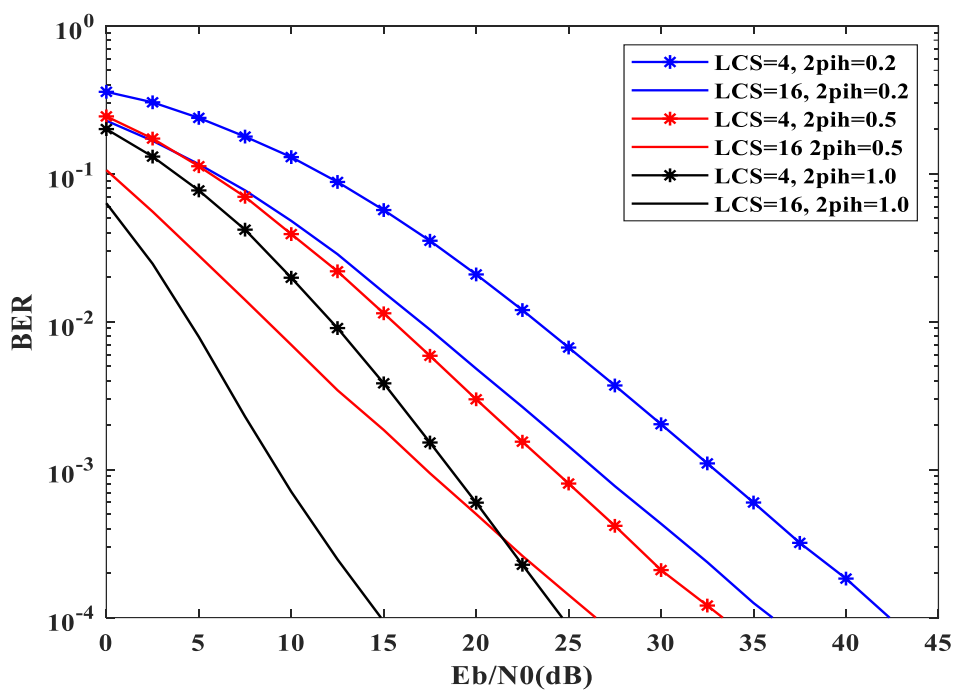

Figure 12. Impact of length of code sequence LCS on the performance of the PM-OFDM-CDMA system.

As discussed above, the CE-OFDM-CDMA system offers high performance, especially at high SNR. On the one hand, the phase modulator in CE-OFDM has the advantage of producing a modulated signal with the lowest possible PAPR. On the other hand, CE-OFDM-CDMA technique can be seen as an extension of MC-CDMA to serve multiple users. A very distinctive feature is that unlike many CDMA multicarrier scheme in the literature, the CE-OFDM-CDMA scheme does not need any reduction in PAPR because the new waveform has a constant power which leads to the lowest possible PAPR. Also, the fact that it combines the CDMA with the orthogonality characteristic of phase transforming subcarriers makes the proposed technique more efficient for a multi-users concept with the advantage of better energy efficiency. 
A comparison with new related works reported in the literature is depicted in Table 2. From this table, we noted that the performance of the PM-OFDM-CDMA system is comparable to other system proposed in the literature.

Table 2. Comparison table.

\begin{tabular}{|c|c|c|c|c|}
\hline References & Modulation technique & Power consumption & Multi-users & complexity \\
\hline Ahmed (2019) & MC-DS-CDMA & High consumption & Yes & High \\
\hline Ansar (2018) & OFDM & High consumption & No & Medium \\
\hline Eeckhaute (2017) & $M C$-CDMA & High consumption & Yes & High \\
\hline El Ghzaoui (2020) & CE-OFDM-MIMO & Les consumption & No & High \\
\hline This work & CE-OFDM-CDMA & Less consumption & Yes & Medium \\
\hline
\end{tabular}

\section{Conclusions}

This work described a phase transformation multiple access schemes. This scheme is based on the principle of CDMA and the OFDM. From the simulation results, we observed that PM-OFDMCDMA is an efficient technique in terms of energy consumption (PAPR = OdB). CE-OFDMCDMA offers high spectral efficiency with low BER due to its low PAPR. In CE-OFDM-CDMA method, the shape of the spectrum varies according to the value of the modulation index $h$. The band occupied by the spectrum increases with the value of $h$. Therefore, based on our results, the CE-OFDM-CDMA system could be considered as a suitable technique for $5 \mathrm{G}$ applications. We showed that the CE-OFDM-CDMA outperforms OFDM-CDMA. But, at low SNR, CE-OFDMCDMA is susceptible to threshold effect due to angle modulation in CE-OFDM.

Topics for further study include studying the effect of channel properties on the performance of the systemas well as, spectral efficiency, the impact of encoding type, and some improvements to further increase system performance.

\section{Conflict of Interest}

The authors approve that there is no conflict of interest to declare for this publication.

\section{Acknowledgments}

The authors sincerely thank the editor, the associate editor and anonymous reviewers for giving this opportunity for our work to be considered for publication.

\section{References}

Ahmed, J. (2019). Spectral efficiency comparison of asynchronous MC-CDMA, MC DS-CDMA and MTCDMA with carrier frequency offset. Arabian Journal for Science and Engineering, 44(3), 1833-1841.

Ansar, H., \& Noor, M.S. (2018). Bandwidth utilization efficiency enhancement for OFDM-based WSN. International Journal of Communication Systems, 31(15). https://doi.org/10.1002/dac.3776.

Baig, I., Farooq, U., Hasan, N.U., Zghaibeh, M., \& Jeoti, V. (2020). A multi-carrier waveform design for 5G and beyond communication systems. Mathematics, 8(9), 1466.

Banelli, P. (2003). Theoretical analysis and performance of OFDM signals in nonlinear fading channels. IEEE Transactions on Wireless Communications, 2(2), 284-293. https://doi.org/10.1109/TWC.2003.808969. 
El Ghzaoui, M., Hmamou, A., Foshi, J., \& Mestoui, J. (2020). Compensation of non-linear distortion effects in MIMO-OFDM systems using constant envelope OFDM for 5G applications. Journal of Circuits, Systems and Computers, 29(16), 2050257.

Gerzaguet, R., Bartzoudis, N., Baltar, L.G., Berg, V., Doré, J.-B., Kténas, D., Font-Bach, O., Mestre, X., Payaró, M., Färber, M., \& Roth, K. (2017). The 5G candidate waveform race: a comparison of complexity and performance. EURASIP Journal on Wireless Communications and Networking, 2017(1), 1-14.

Gupta, M.K., \& Tiwari, S. (2013). Performance evaluation of conventional and wavelet based OFDM system. AEU-International Journal of Electronics and Communications, 67(4), 348-354.

Jangalwa, M., \& Tokekar, V. (2019). Performance analysis of selective mapping and clipping based multicarrier-CDMA system with and without MIMO technique. AEU-International Journal of Electronics and Communications, 101, 62-68.

Judson, D., Devi, T.A.M., Helen, C.N., \& Darwin, P. (2019). Performance analysis of image transmission with different transforms in MC-CDMA. 2019 International Conference on Recent Advances in Energy-Efficient Computing and Communication (ICRAECC), 1-5. Nagercoil, India.

Kumar, T.D., \& Venkatesan, P. (2020). Performance estimation of multicarrier CDMA using adaptive brain storm optimization for $5 \mathrm{G}$ communication system in frequency selective fading channel. Transactions on Emerging Telecommunications Technologies, 31(4), e3829.

Maatouk, A., Çalışkan, E., Koca, M., Assaad, M., Gui, G., \& Sari, H. (2018). Frequency-domain NOMA with two sets of orthogonal signal waveforms. IEEE Communications Letters, 22(5), 906-909.

Mestoui, J., Hmamou, A., Foshi, J., \& El Ghzaoui, M. (2019). BER performance improvement in CEOFDM-CPM system using equalization techniques over frequency-selective channel. Procedia Computer Science, 151, 1016-1021.

Mohammady, S., Farrell, R., Malone, D., \& Dooley, J. (2020). Performance investigation of peak shrinking and interpolating the PAPR reduction technique for LTE-advance and 5G signals. Information, 11(1), 20. https://doi.org/10.3390/info11010020.

Mohammady, S., Farrell, R., Malone, D., \& Dooley, J. (2019). Peak shrinking and interpolating technique for reducing peak to average power ratio. 2019 Wireless Days (WD), 1-6.

Nahar, A.K., Gitaffa, S.A., Ezzaldean, M.M., \& Khleaf, H.K. (2017). FPGA implementation of MCCDMA wireless communication system based on SDR-a review. Review of Information Engineering and Applications, 4(1), 1-19. DOI: 10.18488/journal.79.2017.41.1.19.

Raab, F.H., Asbeck, P., Cripps, S., Kenington, P.B., Popovic, Z.B., Pothecary, N., Sevic, J.F., \& Sokal, N.O. (2002). Power amplifiers and transmitters for RF and microwave. IEEE Transactions on Microwave Theory and Techniques, 50(3), 814-826.

Rajasekaran, A.S., Vameghestahbanati, M., Farsi, M., Yanikomeroglu, H., \& Saeedi, H. (2019). Resource allocation-based PAPR analysis in uplink SCMA-OFDM systems. IEEE Access, 7, 162803-162817.

Razavi, R., Dianati, M., \& Imran, M.A. (2017). Non-orthogonal multiple access (NOMA) for future radio access. In 5G Mobile Communications (pp. 135-163). Springer.

Schaich, F., Wild, T., \& Chen, Y. (2014). Waveform contenders for 5G-suitability for short packet and low latency transmissions. 2014 IEEE 79th Vehicular Technology Conference (VTC Spring), 1-5. Seoul, Korea (South). Doi: 10.1109/VTCSpring.2014.7023145.

Singya, P.K., Kumar, N., \& Bhatia, V. (2017). Mitigating NLD for wireless networks: effect of nonlinear power amplifiers on future wireless communication networks. IEEE Microwave Magazine, 18(5), 7390. 
Taspinar, N., \& Simsir, S. (2020). An efficient SLM technique based on migrating birds optimization algorithm with cyclic bit flipping mechanism for PAPR reduction in UFMC waveform. Physical Communication, 43, 101225.

Thompson, S.C. (2005). Constant envelope OFDM phase modulation (pp. 1619-1619). University of California, San Diego.

Vakilian, V., Wild, T., Schaich, F., Ten Brink, S., \& Frigon, J.F. (2013). Universal-filtered multi-carrier technique for wireless systems beyond LTE. 2013 IEEE Globecom Workshops (GC Wkshps), 223-228.

Van Eeckhaute, M., Bourdoux, A., De Doncker, P., \& Horlin, F. (2017). Performance of emerging multicarrier waveforms for 5G asynchronous communications. EURASIP Journal on Wireless Communications and Networking, 2017(1), 29. 